$\mu \mathrm{g}$ budesonide or equivalent) received once-daily tioR $5 \mu \mathrm{g}$ or placebo Respimat ${ }^{\circledR}$. In two 24-week trials (MezzoTinA-asthma ${ }^{\circledR}$ : NCT01172808/NCT01172821), patients on ICS (400-800 $\mu \mathrm{g}$ budesonide or equivalent) received once-daily tioR $5 \mu \mathrm{g}$ or $2.5 \mu \mathrm{g}$, twice-daily salmeterol $50 \mu \mathrm{g}$ via hydrofluoroalkane metered-dose inhaler (active comparator) or placebo (identical devices in a double-dummy protocol). Pre-planned analyses (pooled data) of time to first severe exacerbation and time to first episode of asthma worsening were performed in $\mathrm{T}_{\mathrm{H}}$ 2-low and $\mathrm{T}_{\mathrm{H}}$ 2-high subgroups: total serum immunoglobulin $(\mathrm{IgE}) \leq$ or $>430 \mu \mathrm{g} / \mathrm{L}(179.2 \mathrm{IU} / \mathrm{L})$; blood eosinophils $\leq$ or $>0.6 \times 10^{9} / \mathrm{L}(600 / \mu \mathrm{L})$.

Results 912 patients with severe asthma received tioR $5 \mu \mathrm{g}$ or placebo Respimat ${ }^{\circledR}:$ 205/182 were reported with $\operatorname{IgE}>430 \mu \mathrm{g} / \mathrm{L}$ and $99 / 87$ with an eosinophil count of $>0.6 \times 10^{9} /$ L. 2100 patients with moderate asthma received tioR $5 \mu \mathrm{g}$ or $2.5 \mu \mathrm{g}$, salmeterol or placebo: 319/320/319/326 were reported with $\operatorname{IgE}$ $>430 \mu \mathrm{g} / \mathrm{L}$ and 104/103/111/107 with an eosinophil count of $>0.6 \times 10^{9} / \mathrm{L}$. Time to first severe exacerbation was longer with tioR versus placebo (Table 1 ) in patients with severe or moderate asthma, independent of $\operatorname{IgE}$ and eosinophils (interaction $\mathrm{p}$ values [Cox regression] 0.169 and 0.754, respectively, for PrimoTinAasthma $^{\circledR}$; analyses not performed for MezzoTinA-asthma ${ }^{\circledR}$ because of low incidence of severe exacerbations). Time to first asthma worsening was longer with tioR versus placebo (Table 1) in patients with moderate or severe asthma, independent of $\operatorname{IgE}$ (interaction $\mathrm{p}$ values 0.998 [PrimoTinA-asthma ${ }^{\circledR}$ ] and 0.041 [MezzoTinA-asthma ${ }^{\circledR}$ ]) and eosinophils (interaction $\mathrm{p}$ values 0.251 [PrimoTinA-asthma ${ }^{\circledR}$ ] and 0.125 [MezzoTinA-asthma ${ }^{\circledR}$ ]).

\begin{tabular}{|c|c|c|c|c|}
\hline & \multicolumn{4}{|c|}{$\begin{array}{l}\text { All comparisons versus placebo } \text { Respimat }^{\oplus} \text { or } \\
\text { placebo HFA-MDI, hazard ratio }\end{array}$} \\
\hline & \multicolumn{2}{|c|}{ Serum $\lg E(\mu g / L)$} & \multicolumn{2}{|c|}{ Eosinophils $\left(\times 10^{9} / \mathrm{L}\right)$} \\
\hline & $\leq 430$ & $>430$ & $\leq 0.6$ & $>0.6$ \\
\hline \multicolumn{5}{|c|}{ Time to first severe asthma exacerbation } \\
\hline \multicolumn{5}{|c|}{ PrimoTinA-asthma ${ }^{\oplus}$ (ICS + LABA) } \\
\hline TioR $5 \mu \mathrm{g}$ & 0.75 & 1.07 & 0.81 & 0.75 \\
\hline QD & $p=0.162$ & $p=0.692$ & $p=0.162$ & $p=0.218$ \\
\hline \multicolumn{5}{|c|}{ MezzoTinA-asthma (ICS) } \\
\hline TioR $5 \mu \mathrm{g}$ & 0.86 & 0.61 & 0.65 & 1.04 \\
\hline$Q D^{b}$ & $p=0.691$ & $p=0.107$ & $\mathrm{p}=0.099$ & $p=0.953$ \\
\hline TioR $2.5 \mu \mathrm{g}$ & 0.51 & 0.50 & 0.41 & 1.25 \\
\hline$Q D^{b}$ & $p=0.119$ & $p=0.033$ & $p=0.003$ & $p=0.715$ \\
\hline \multirow[t]{2}{*}{ Salmeterol ${ }^{\mathrm{c}}$} & 0.82 & 0.68 & 0.62 & 1.76 \\
\hline & $p=0.594$ & $p=0.189$ & $p=0.066$ & $p=0.310$ \\
\hline \multicolumn{5}{|c|}{ Time to first episode of asthma worsening ${ }^{a}$} \\
\hline \multicolumn{5}{|c|}{ PrimoTinA-asthma $($ ICS + LABA) } \\
\hline TioR $5 \mu \mathrm{g}$ & 0.73 & 0.73 & 0.65 & 0.85 \\
\hline QD & $p=0.030$ & $p=0.017$ & $\mathrm{p}<0.001$ & $p=0.360$ \\
\hline \multicolumn{5}{|c|}{ MezzoTinA-asthma (ICS) } \\
\hline TioR $5 \mu \mathrm{g}$ & 0.88 & 0.83 & 0.90 & 0.70 \\
\hline$Q D^{b}$ & $\mathrm{p}=0.495$ & $p=0.220$ & $p=0.410$ & $p=0.170$ \\
\hline TioR $2.5 \mu \mathrm{g}$ & 0.45 & 0.81 & 0.60 & 0.91 \\
\hline$Q D^{b}$ & $\mathrm{p}<0.001$ & $p=0.157$ & $\mathrm{p}<0.001$ & $p=0.691$ \\
\hline \multirow[t]{2}{*}{ Salmeterol $^{\mathrm{C}}$} & 0.60 & 0.84 & 0.71 & 0.92 \\
\hline & $p=0.009$ & $p=0.231$ & $p=0.009$ & $p=0.714$ \\
\hline
\end{tabular}

${ }^{a}$ Defined as either a progressive increase in symptoms or a decline of $\geq 30 \%$ in best morn-

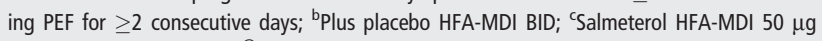
BID plus placebo Respimat ${ }^{\circledR}$ QD.

$B I D$, twice-daily; HFA-MDI, hydrofluoroalkane metered-dose inhaler; QD, once-daily; tioR, tiotropium Respimat ${ }^{\oplus}$.
Conclusion Once-daily tiotropium Respimat ${ }^{\circledR}$ add-on to at least ICS reduced the risk of severe exacerbation and asthma worsening in patients with moderate or severe symptomatic asthma, independent of $\mathrm{T}_{\mathrm{H}}^{2}$ phenotype.

\section{P152 FLUTICASONE FUROATE (FF)/VILANTEROL (VI) ONCE DAILY REDUCES ASTHMA SYMPTOMS BOTH DAY AND NIGHT}

${ }^{1}$ DA Leather, ${ }^{2} \mathrm{R}$ Forth, ${ }^{1} \mathrm{~L}$ Yates, ${ }^{3} \mathrm{LA}$ Jacques. ${ }^{1}$ GlaxoSmithKline Respiratory Global Franchise, London, UK; ${ }^{2}$ PAREXEL International Research Triangle Park, Durham, USA; ${ }^{3}$ Respiratory Medicines Development Centre, GlaxoSmithKline, London, UK

\subsection{6/thoraxjnl-2015-207770.289}

Introduction and objectives FF/VI is the first once daily inhaled corticosteroid/long-acting $b_{2}$-agonist combination available for the treatment of asthma. Results from five phase III studies that have previously been presented demonstrated a sustained $24 \mathrm{~h}$ improvement in lung function and improvement in symptomfree $24 \mathrm{~h}$ periods.

Methods Post-hoc analyses of diary card data from these studies were performed to examine whether there was any difference in the contribution of the day and night time symptom-free period to the $24 \mathrm{~h}$ symptom-free period. The diary card scale used is described below.

Day-time Symptom Score:

$0=$ No symptoms during the day

1 = Symptoms for one short period during the day

$2=$ Symptoms for two or more short periods during the day

3 = Symptoms for most of the day which did not affect my normal daily activities

4 = Symptoms for most of the day which did affect my normal daily activities

5 = Symptoms so severe that I could not go to work or perform normal daily activities

Night-time Symptom Score:

$0=$ No symptoms during the night

$1=$ Symptoms causing me to wake once (or wake early)

2 = Symptoms causing me to wake twice or more (including waking early)

3 = Symptoms causing me to be awake for most of the night

4 = Symptoms so severe that I did not sleep at all

To be counted as symptom-free during the day or night the patient needed to record a score of 0 .

Results The post-hoc analyses demonstrated that the improvements in day and night time symptom -free periods were similar to the $24 \mathrm{~h}$ symptom free periods. See Figure 1 below.

Conclusions In general benefits in symptom free days and symptom free nights contributed to the benefit of FF/VI over comparator groups in terms of $24 \mathrm{~h}$ symptom free periods.

\section{P153 FLUTICASONE PROPIONATE/FORMOTEROL PRESSURISED METERED-DOSE INHALER '2-3-4' TRAINING PARADIGM AIDS CORRECT INHALER TECHNIQUE}

${ }^{1} \mathrm{D}$ Bell, ${ }^{2} \mathrm{~L}$ Mansfield, ${ }^{2} \mathrm{M}$ Lomax, ${ }^{2} \mathrm{~S}$ Dissanayake. ${ }^{1}$ Bio-Kinetic Europe Limited, Belfast, UK; ${ }^{2}$ Mundipharma Research Limited, Cambridge, UK

\subsection{6/thoraxjnl-2015-207770.290}

Background Inhaler technique is crucial to effective disease control. Amongst the most frequent mistakes made with all inhalers are the failure to exhale adequately, an insufficient breath-hold 
and, with pressurised metered-dose inhalers (pMDIs), the failure to inhale "slowly". We present an easy to remember '2-3-4' training paradigm, designed to address these errors, that was used in an open-label, cross-over study comparing patient handling of fluticasone propionate/formoterol (FP/FORM; flutiform $^{\circledR}$ ) breath-actuated inhaler (BAI) and pMDI devices. Here we present the pMDI data.

Methods The study was carried out in 311 patients ( $\geq 12$ years) with persistent asthma (64\%), COPD (28\%), or both asthma and COPD ( $8 \%$ of patients). Patients were randomised to one of two sequences $(\mathrm{BAI} / \mathrm{pMDI}$ or $\mathrm{pMDI} / \mathrm{BAI})$. Patients were trained to correctly use each device prior to an assessment of correct handling according to prespecified criteria. Eight steps were assessed including, but not limited to: exhale $\geq 2 \mathrm{~s}$; inhale $\geq 3 \mathrm{~s}$; hold breath $\geq 4 \mathrm{~s}$ : the 2-3-4 technique. Patients were assessed on a single device (BAI or pMDI) at each visit, with 7-21 days between the two visits.

Results Overall, $77.2 \%$ of patients demonstrated correct performance of all steps of FP/FORM pMDI use at their first attempt post-training $(\mathrm{N}=307)$; a further $14.0 \%$ required 2 attempts. $99.3 \%$ of patients were successfully trained within 15 min. Step 2 (Removes cap) and steps 4-6 (Places upright in mouth; starts inhaling and actuates; inhales for at least 3 s) were considered critical steps, and $82.4 \%$ of patients demonstrated correct performance of all 4 critical steps at their first attempt post-training. $96.1 \%, 87.6 \%$ and $95.8 \%$ of patients correctly followed each component of the '2-3-4' training paradigm with the pMDI at the first attempt post-training (exhale $\geq 2 \mathrm{~s}$, inhale $\geq 3 \mathrm{~s}$; hold breath $\geq 4 \mathrm{~s}$, respectively).

Conclusions Almost all patients can be rapidly taught to use a pMDI (within $15 \mathrm{~min}$ ) using a simple training method suitable for all clinical settings. An easy to remember, sequential "2-3-4" paradigm is effective in encouraging patients to exhale to residual volume, to inhale slowly, and to breath hold satisfactorily: manoeuvres that are frequently performed incorrectly without such instruction.

Sponsor Mundipharma Research Ltd.

\section{P154}

EVALUATION OF INHALER TECHNIQUE MASTERY FOR BUDESONIDE FORMOTEROL SPIROMAX ${ }^{\circledR}$ COMPARED WITH SYMBICORT TURBOHALER ${ }^{\circledR}$ IN ADULT PATIENTS WITH ASTHMA: PRIMARY RESULTS FROM THE EASY LOW INSTRUCTION OVER TIME [ELIOT] STUDY

${ }^{1} \mathrm{H}$ Chrystyn, ${ }^{2} \mathrm{R}$ Dekhuijzen, ${ }^{3} \mathrm{C}$ Rand, ${ }^{4} \mathrm{~S}$ Bosnic-Anticevich, ${ }^{5} \mathrm{~N}$ Roche, ${ }^{6} \mathrm{~F}$ Lavorini, ${ }^{7} \mathrm{~V}$ Thomas, 7 J Steele, ${ }^{7} \mathrm{P}$ Raju, ${ }^{7} \mathrm{D}$ Freeman, ${ }^{7}$ I Small, ${ }^{8} \mathrm{~J}$ Canvin, ${ }^{9} \mathrm{D}$ Price. ${ }^{1}$ University of Plymouth, Plymouth, UK; ${ }^{2}$ University Nijmegen Medical Centre, Nijmegen, The Netherlands; ${ }^{3}$ Johns Hopkins School of Medicine, Baltimore, USA; ${ }^{4}$ University of Sydney, Sydney, Australia; ${ }^{5}$ University Paris Descartes, Paris, France; ${ }^{6}$ University of Florence, Florence, Italy; ${ }^{7}$ Research in Real Life Ltd., Cambridge, UK; ${ }^{8}$ Teva Branded Pharmaceutical Products R\&D, Inc., Pennsylvania, USA; ${ }^{9}$ Observational and Pragmatic Research Institute Pte Ltd, Singapore, Singapore

\subsection{6/thoraxjnl-2015-207770.291}

Introduction and objectives Technical errors in the use of inhalers are associated with poor asthma control. This study evaluated achievement of mastery in a training environment using a randomised cross-over design (stage 1), followed by randomization into a prospective 12 -week trial to assess maintenance of mastery in patients receiving inhaled corticosteroids (ICS)/long-acting $\beta_{2}$-agonists (LABA) via SPIROMAX versus ICS/ LABA received via TURBOHALER (stage 2).

Methods Patients with asthma were randomised to a 6-step training protocol using empty Spiromax and empty Turbohaler devices. The proportion of patients achieving and maintaining inhaler mastery, respectively defined as the absence of health care professional (HCP)-observed errors by training step 3 (instructional video) in stage 1 , and the absence of HCPobserved errors after 12 weeks of inhaler use in stage 2 , were analysed using logistic regression. The maintenance of independent expert video-observed inhaler mastery was analysed using logistic regression. Total observed errors (HCP and technology) were analysed using a negative binomial regression model. Vitalograph Pneumatic Spirometry results were compared using a Mann Whitney U test.

Results A total of 493 (89.1\%) patients (stage 1) and 305 (61.9\%) (stage 2) were eligible for the full analysis set. The odds of maintaining inhaler mastery were not significantly different

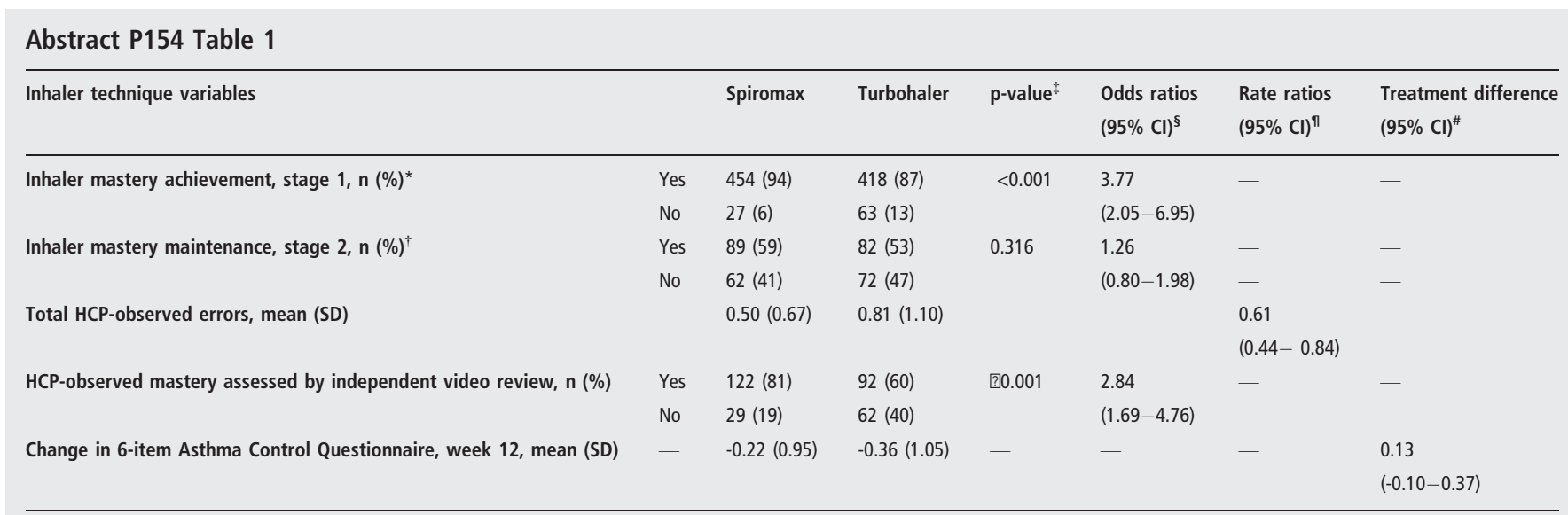

*In stage 1, 493 patients were randomly assigned to either empty Spiromax followed by empty Turbohaler or empty Turbohaler followed by empty Spiromax. Achieving inhaler mastery was defined as the absence of HCP-observed errors by the end of step 3 of a 6 -step standardised inhaler training protocol for empty Spiromax compared to empty Turbohaler inhalers; ${ }^{\dagger}$ In stage 2 . 305 out of 395 patients $(61.9 \%)$ patients were eligible for the full analysis set. Maintaining inhaler mastery was defined as the absence of HCP-observed errors after 12 weeks of inhaler use; ${ }^{\ddagger}$ The $\mathrm{p}$-value for the treatment comparison is based on chi-square. $\mathrm{p}<0.05$ considered statistically significant; ${ }^{\S}$ Logistic regression; ${ }^{9}$ Negative binomial regression; ${ }^{\#}$ Analysis of variance; Cl: confidence interval; HCP: Health care professional; SD, standard deviation. 